\title{
Recurrent Ovarian Cancer presenting as Bilateral Internal Jugular Venous Thrombosis - A Case Report
}

\author{
Manzoor Ahmad Banday, M.D., ' Feroze Ahmad Shaheen, M.D., ${ }^{2}$ Syed Nisar Ahmad, M.D.' \\ Departments of 'Medical Oncology, and ${ }^{2}$ Radiodiagnosis, Sher-i-Kashmir Institute of Medical Sciences Srinagar
}

\section{A B S T R A C T}

Venous thrombosis as an initial manifestation of an underlying malignancy is rare but well known. Infact, the index of suspicion is so high that spontaneous venous thrombosis in a patient warrants screening for an occult cancer. Bilateral internal jugular venous thrombosis is rarer among the sites of idiopathic venous thromboembolism. Besides carrying risk for death due to pulmonary embolism, as a spontaneous occurrence, it strongly points to underlying malignancy when other causes have been ruled out. JMS 2012;15(1):72-73.

KeyWords: Venous thrombosis, malignancy, ca ovary, relapse

Bilateral internal jugular venous (IJV) thrombosis most often results from central venous catheters, hypercoagulability states, congestive heart failure etc. Idiopathic IJV thrombosis may be initial manifestation of an occult malignancy. We present a case of recurrent ovarian carcinoma whose initial manifestation was bilateral IJV thrombosis.

\section{Case report}

45 year old female had been diagnosed as ovarian cancer one year back and was currently under remission for last six months. The patient presented to for follow-up to the oncology clinic with bilateral painless neck swelling. Clinical examination revealed engorged neck veins on both sides with large cord like masses on both sides. Ultrasonography of neck with color Doppler revealed bilateral internal jugular venous thrombosis with enlarged collateral veins in the neck (Figures

\section{Correspondence:}

Dr. Manzoor Ahmad Banday

Department of Medical Oncology

Sher-i-Kashmir Institute of Medical Sciences, Srinagar, Kashmir, India, 190011

Phone: 0194-240-0348 Ext. 2120, Fax: 0194-240-3470

E-Mail: mutaibma@yahoo.com
1A, 1B, 1C). There was no associated thrombosis of subclavian veins on either side. Routine hematological indices and coagulation profile were normal. Contrast CT neck revealed bilateral internal jugular venous thrombosis without extension into superior vena cava (SVC). (Figure 2A, 2B). Cross sectional imaging of abdomen and pelvis revealed recurrent ovarian mass with moderate ascites. Tumor marker CA 125 was markedly raised.

\section{Discussion}

Association of idiopathic thromboembolism and occult malignancy is a known fact with a serious debate on the need to screen the patients with idiopathic thromboembolism for cancer. ${ }^{1}$ There is a 3-19 fold increase in the prevalence of concomitant cancer in patients with secondary and idiopathic venous thromboembolism respectively. ${ }^{2}$

Upper extremity deep vein thrombosis (UEDVTS) accounts for $4 \%$ of all deep vein thrombosis (DVT) and $20 \%$ of UEDVTS are apparently spontaneous. ${ }^{3}$ The morbidity and mortality from UEDVTS at 1,3 and 12 months were 13\%, $31 \%$ and $40 \%$ for subclavian and axillary thrombosis, $14 \%$, $33 \%$ and $42 \%$ for IJV thrombosis and $23 \%, 44 \%$ and $55 \%$ for combined subclavian and IJV thrombosis. ${ }^{4}$ Different types of cancers ranging from gastrointestinal to cancers of prostate 


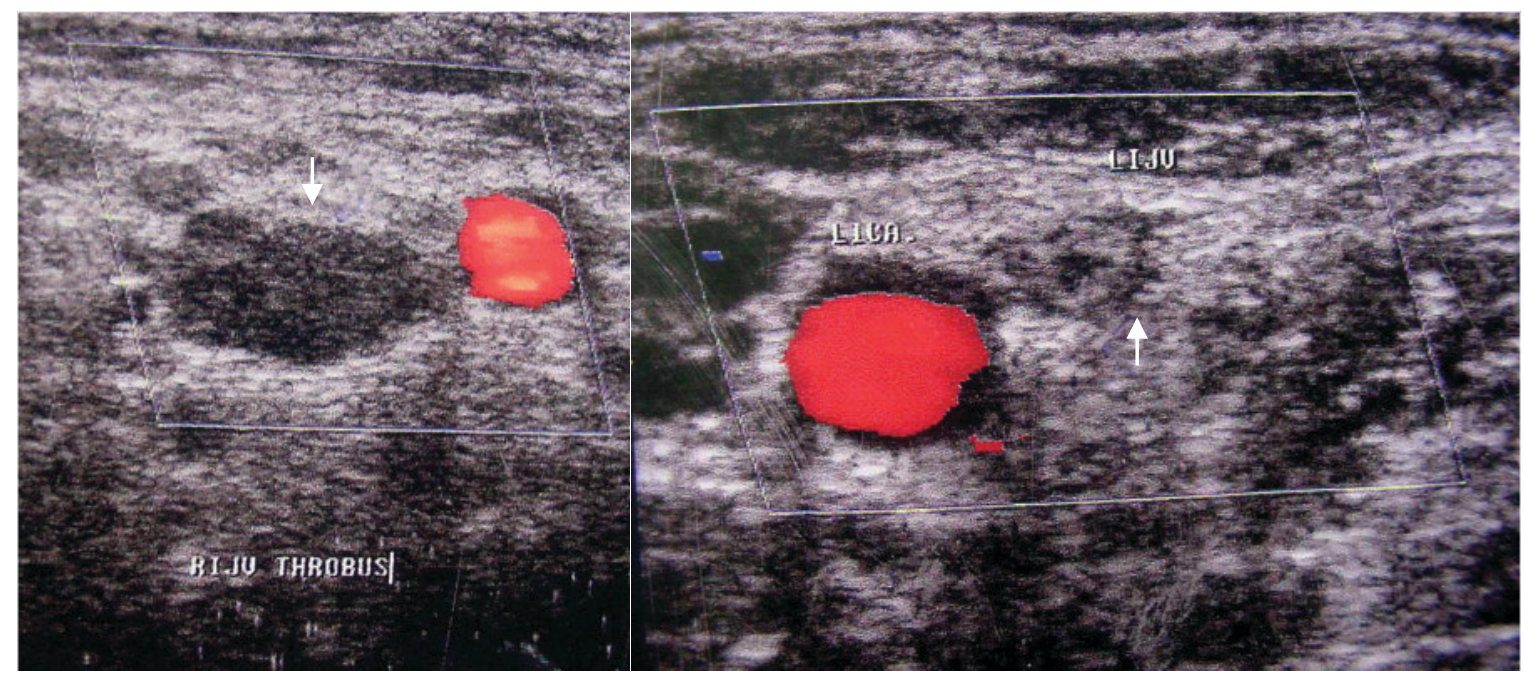

FIGURES IA, 1B: Doppler Scan showing large organized thrombus in RT. and LT. IJVs with normal color signal from RT. Common Carotid Artery

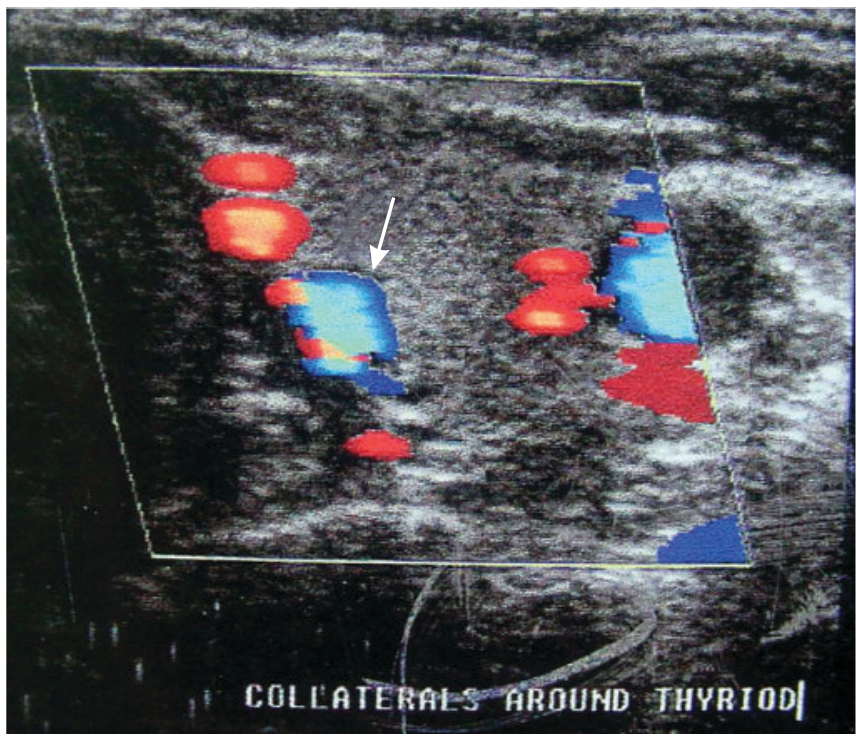

FIGURE IC: Collateral veins along Lt Lobe of Thyroid bypassing LT. IJV

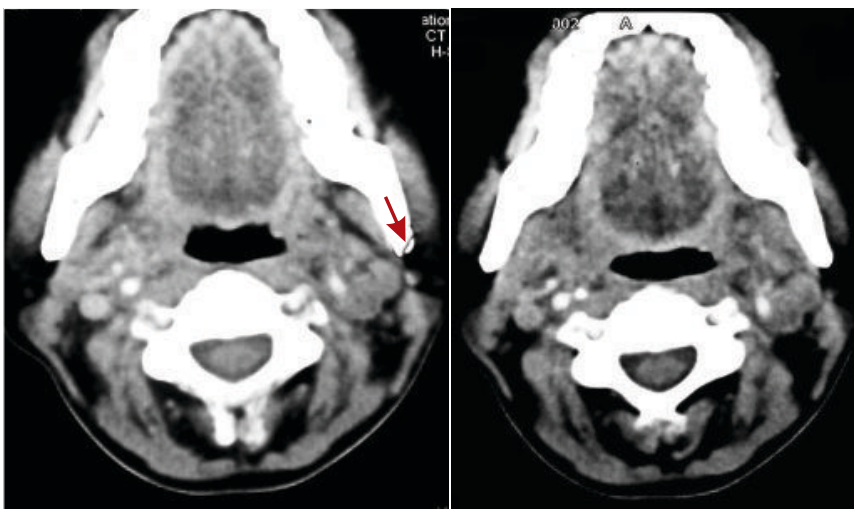

FIGURES 2A \& 2B: Post contrast CT axial scans showed enlarged Internal jugular veins (IJVs) with filling defects in the lumen.(arrow)

and ovary are known to present as IJV thrombosis. Thus IJV thrombosis represents an epiphenomenon of underlying occult cancers.
The prevalence of occult cancer in patients with idiopathic VTE is $4-10 \%$, however the prevalence rate varies depending upon the depth of routine examination, difference in threshold for suspicion besides other things.

In our case the recurrence of the ovarian cancer became first manifest as bilateral IJV thrombosis. The patient had undergone remission 6 months back and was on follow-up. As mentioned earlier routine hematological investigations besides assays for antinuclear antibodies were negative. This case illustrates the close association between idiopathic VTE and recurrent cancer. Although many studies have been conducted and epidemiological association established, no definite causative relation has yet been discovered between the two. This case also highlights the need for a high index of suspicion of new or recurrent cancer in patients presenting with idiopathic DVT.

\section{References}

1. Piccioli A, Prandoni P. Venous thromboembolism as first manifestation of cancer. Acta Haematol 2001; 106:13-7.

2. Otten HM, Prins MH. Venous thromboembolism and occult malignancy. Thromb Res 2001;102(6):V187-94.

3. Bernardi E, Piccioli A, Marchiori A, Girolani B, Prandoni P. Upper extremity deep vein thrombosis: risk factors, diagnosis and management. Semin Vasc Med 2001;1(1): 105-10.

4. Ascher E, Salles-Cunha S, Hingorani A. Morbidity and mortality associated with internal jugular vein thrombosis. Vasc Endovascular Surg 2005; 39(4):335-9.

5. Thomas S, Sawhney S, Kapur BM. Bilateral massive internal jugular vein thrombosis in carcinoma of thyroidCT evaluation. Clin Radiol 1991;43(6):433-4.

6. Erkoc R, Uzun K, Yuca K, Etlik O, Dogan A. Internal jugular vein thrombosis - Two different etiologies. European Journal of General Medicine 2005;2(3):123-28. 